\title{
MALDI mass spectrometry imaging in oncology (Review)
}

\author{
TIMO GEMOLL, UWE J. ROBLICK and JENS K. HABERMANN \\ Department of Surgery, Laboratory for Surgical Research, University of Lübeck, D-23538 Lübeck, Germany
}

Received April 4, 2011; Accepted August 9, 2011

DOI: $10.3892 / \mathrm{mmr} .2011 .566$

\begin{abstract}
Matrix-assisted laser desorption/ionization (MALDI) mass spectrometry imaging (MSI) has improved over the years and is increasingly being used for biomarker discovery directly from human tissue sections. State-of-the-art technology currently enables a resolution down to $20 \mu \mathrm{m}$. MSI therefore allows the correlation of spatial and temporal protein expression profiles with distinct morphological features without requiring target-specific reagents, such as antibodies. Several studies have demonstrated the strength of the technology for uncovering new markers that correlate with disease severity as well as prognosis and therapeutic response. This review provides an overview of MALDI imaging functionality and its advantages and disadvantages, and provides a current literature overview of malignancy-based biomarker detection. Further improvements on instrumentation sensitivity, image processing and sample preparation will enable the detection of novel, tissue-specific biomarkers. However, emphasis should be given to large validation studies and/or subsequent identification of differentially observed protein peaks in order to transfer MSI protein profiling and/or novel biomarkers thereof into clinical use.
\end{abstract}

\section{Contents}

1. Introduction

2. MALDI-TOF imaging technology

3. Data collection

4. Current applications of MALDI-TOF MS imaging technology for tissue proteomics of various cancer entities

5. MALDI-TOF imaging data relating to cancer

6. Conclusion

\section{Introduction}

The term 'proteome' was first defined in 1994 and defines the entirety of proteins expressed by the genome. While DNA

Correspondence to: Dr Jens K. Habermann, Department of Surgery, Laboratory for Surgical Research, University of Lübeck, Ratzeburger Allee 160, D-23538 Lübeck, Germany

E-mail: jens.habermann@gmail.com

Key words: biomarker, imaging mass spectrometry, proteomics, cancer acts like a 'blueprint', proteins are the dynamic components of the cell. Proteomics is therefore understood as the consecutive step following genomics. Proteomics techniques have rapidly evolved, however, clinical implementations have rarely been successful (1). This may be due to several causes: Firstly, proteomics techniques, such as two-dimensional gel electrophoresis (2-DE) are too time-consuming for routine clinical use and only allow relative quantification (2). Secondly, clinical samples are too complex and underrepresented cells, compartments or proteins are difficult to access (3). Thirdly, protein biomarkers may hamper reproducible analyses due to post-translational modifications or stability issues (4). Furthermore, large validation studies using standardized sample collections to bring potential novel biomarkers into the clinic are often lacking. Against this background, personalized medicine still depends on the introduction of high-throughput, quantitative and sensitive proteomic approaches in order to substantially improve individual diagnosis, prognosis, therapy monitoring and surveillance.

Understanding the modification of the proteome in the presence of disease is the goal of 'clinical proteomics'. This may be achieved by several approaches. Until recently, twodimensional polyacrylamide gel electrophoresis (2-DE-PAGE) has dominated the field. In 2-DE, proteins are separated first by their isoelectric point, followed by a separation based on molecular weight (5). The combination of these two orthogonal separation techniques resolves proteins into spots, generating a map that can be considered as the 'protein fingerprint' of that sample. While the resolution of complex protein mixtures obtained with 2-DE is far superior to that of conventional one-dimensional protein electrophoresis, current 2-DE methods have several technical disadvantages that limit their widespread application. Other techniques used for the expression analysis of proteins are surface-enhanced laser desorption/ ionization time-of-flight mass spectrometry (SELDI-TOF-MS), liquid-chromatography combined with MS (LC-MS), and more quantitative methods, such as isotope-coded affinity tags and isotope tags for relative and absolute quantification (6-12). The advantages and disadvantages of these methods are summarized in Table I.

Recently, matrix-assisted laser desorption/ionization (MALDI) mass spectrometry imaging (MSI) has been increasingly applied for cancer proteomics. MSI allows the elucidation of spatial and temporal protein expression profiles directly from tissue sections with a resolution down to $20 \mu \mathrm{m}$. MSI therefore overcomes the restrictions by proteome analyses of whole tissue extracts. In addition, MSI allows the direct correlation 
Table I. Advantages and disadvantages of selected proteomic technologies for protein profiling.

Technology Advantages Disadvantages

MALDI-TOF MS

imaging (MSI)

SELDI-TOF MS

MALDI-TOF MS

LC-MS

2-DE PAGE

2-DE DIGE

Protein chips

ICAT

Protein microarrays
Correlation between protein expression

and tissue histology

High throughput of protein peaks per sample

High throughput

Direct application of whole sample

Access to PMT

Small amount of sample needed

High throughput

Access to PMT

Determination of molecular weight

and amino acid sequence

Direct identification

Resolution up to 1000 proteins

Access to PMT

High resolution of proteins

Detection of PTM

Direct comparison of samples in one gel

(enhanced reproducibility)

Higher sensitivity

High throughput

Easily scalable

Sensitive and quantitative method

Direct identification

High throughput

Direct identification
Unsuitable for high molecular weight proteins

Requires sequence database for identification High costs of equipment purchase

Time-consuming

No direct identification

Unsuitable for high molecular weight proteins

Lower resolution and mass accuracy

than MALDI-TOF

Limited to detection of bound proteins

Sample fractionation required

High costs of equipment purchase

Need of sequence database for identification

Unsuitable for high molecular weight proteins

Low throughput

High costs of equipment purchase

Time-consuming

Complicated comparison of different samples

Large amount of sample needed

Poor resolution of extreme masses

Poor resolution of extremely acidic

and basic proteins

No direct protein identification

See 2-DE PAGE

Not yet standardized

Cross-reactivity

Low throughput

Cystein residue must be present for labeling

High costs of equipment purchase

Synthesis of different probes

Cross-reactivity of protein expression with distinct tissue morphology. The high resolution and correlation with histomorphology as well as the increased speed of data acquisition have elicited high expectations for MSI's clinical applicability.

This review focuses on recent advances in MSI technology and surveys current MSI-based studies analyzing tissue proteins for their potential clinical value for early diagnosis, prognosis and prediction in cancer.

\section{MALDI-TOF imaging technology}

This image technology utilizing mass spectrometry is based on MALDI time-of-flight mass spectrometry (MALDI-TOF-MS), comprising a MALDI plate and MS instrumentation, as well as the associated software.
The MALDI MSI technology allows the reconstruction of molecular images based on the spatial distribution of molecules in tissues. A tissue section is treated with a matrix and scanned in a MALDI mass spectrometer (Fig. 1). As the majority of tissues with correlating clinical follow-up data are collected and stored as formalin-fixed paraffin-embedded (FFPE) samples in pathologists' archives, it is essential that MSI can be applied to FFPE and formalin-fixed tissues. Until recently, FFPE samples were believed to be unusable for proteomics approaches due to protein cross-linking caused by formalin fixation (13). However, use of antigen retrieval techniques coupled with in situ tryptic digestion has allowed the analysis of FFPE samples by MSI (14). Mass spectrometric data are acquired by performing a raster of the sample by the laser beam with a predefined number of laser shots per grid 
coordinate. Each spot produces a mass spectrum obtained from proteins present within the irradiated area. Detected proteins/ions are displayed as a series of peaks that are referred to as the protein fingerprint or ion signature. The position of an individual protein/ion in the spectrum corresponds to its time-of-flight. Following computer-based normalization, peak intensity is proportional to the amount of sample-derived protein derived from the tissue. All acquired mass spectra from the entire tissue are then compiled to create a 2-DE map. This map may then be compared with those from other tissue samples to identify changes in protein or peptide expression. Alternatively, comparisons of the maps from various areas of the same tissue section may be performed as well. A marked advantage of this technology is the high-throughput discovery of protein markers, since the expression profiles of numerous proteins can be obtained without the need for antibodies. In addition, correlations between protein expression and tissue histology can also be studied easily, since the expression profiles are obtained directly from the tissue sample.

In addition to the above-mentioned advantages of MSI technology, it is also of crucial significance to be aware of its limitations. One difficulty that has been addressed is the direct identification of biomarkers in tissues. Bottom-up strategies using on-tissue trypsin digestion have been developed for frozen $(15)$ and FFPE $(16,17)$ tissues. Here, time-consuming processing for purification and identification of the proteins is required beyond the MALDI-TOF experiment. In contrast, the ability to perform on-tissue top-down protein characterization would be ideal as a future perspective.

A further purpose lays in the three-dimensional reconstruction to obtain tumor maps. It is well known that a tumor comprises distinct hierarchies reflected by distinguished cell types at various developmental stages. Furthermore, invading and/or metastasizing cancer cells are detectable in varying densities within different tissue types and are influenced by their surrounding environment (e.g., connective tissue and micro-environmental factors). Specific regions of tumors, such as the invasion front, differ functionally from other regions, and hypoxic regions inside tumors may have a completely different metabolism. In conclusion, a tumor is a highly complex system at the cellular level (18). Therefore, MSI-based cancer proteomics would benefit substantially if its two-dimensionality could be scaled up for three-dimensional measures. Consistent with this, the maximum resolution of $20 \mu \mathrm{m}$ does not allow the characterization of individual cells. The development of increased or super-resolution down to single cells would therefore be of high benefit for molecular-pathological research (19).

\section{Data collection}

The PubMed database was searched in order to identify relevant studies based on the terms 'cancer', 'MALDI' and 'imaging'. Each search was limited to studies on humans published in English. In addition, bibliographies of articles were further examined for relevant citations. The initial search obtained the following hits: i) Results for 'MALDI' and 'imaging', 572 (70 reviews); ii) results for 'cancer', 'MALDI' and 'imaging', 118 (14 reviews); and iii) results for 'cancer', 'MALDI', 'imaging' and 'biomarker', 42 (6 reviews).

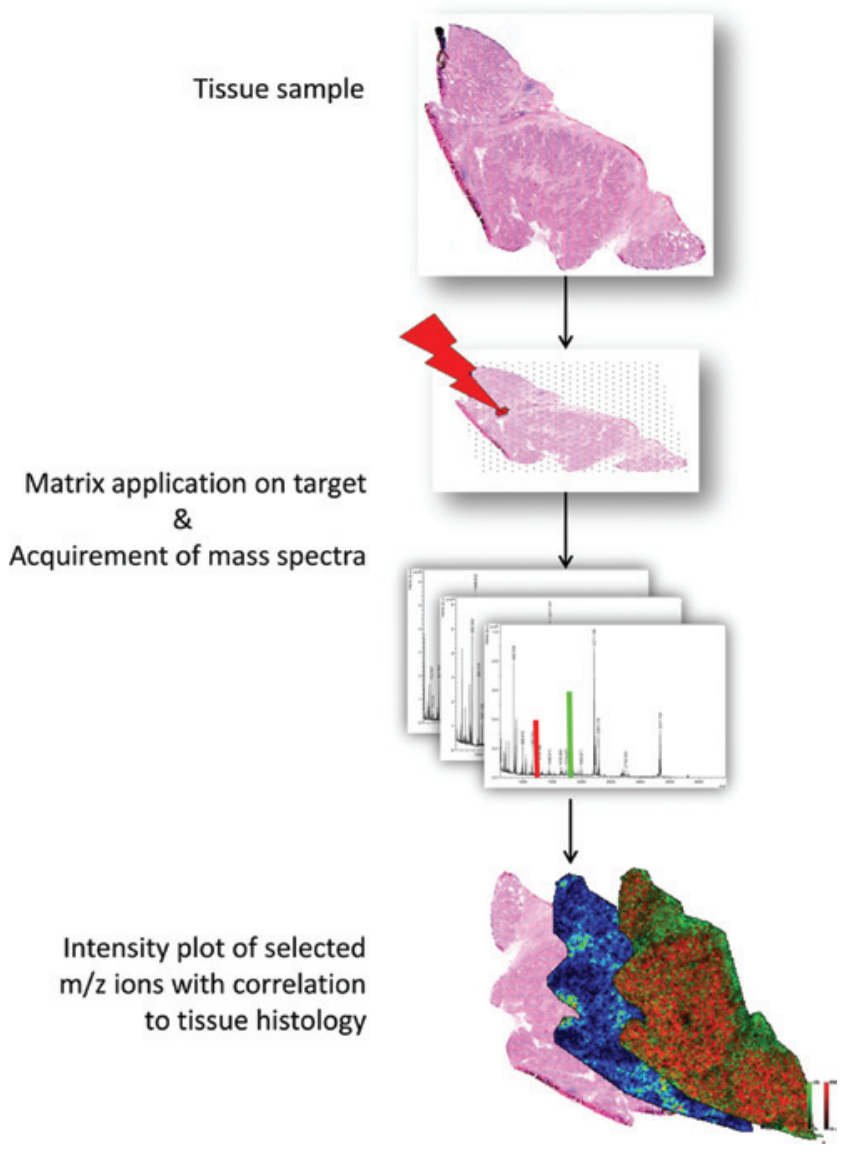

Figure 1. Schematic workflow of a typical MSI experiment.

Since this review focuses on MALDI-TOF-based imaging for biomarker detection in cancer, all 42 publications covering these search terms simultaneously were considered. These were further down-selected based on the following requirements: Only studies dealing with protein profiling examining cases and controls were included, allowing the determination of sensitivity and specificity. Publications with information regarding storing temperature and training and validation set were preferably selected due to a higher degree of comparability. In total, 7 studies fulfilled the requirements and were included in this review.

\section{Current applications of MALDI-TOF MS imaging technology for tissue proteomics of various cancer entities}

Numerous articles concerning proteomics studies have been published regarding various techniques in clinical cancer proteomics. However, only a small number of these have examined MALDI-TOF MS imaging applications in cancer research (Table II).

One of the first studies was carried out on prostate cancer in 2007 by Schwamborn et al, who evaluated 22 tissue samples from patients undergoing a radical prostatectomy (20). The samples were stored at $-80^{\circ} \mathrm{C}$. The cancer group consisted of 11 samples from prostate cancer patients at various clinical stages: Gleason score $6(n=4), 7(n=4)$ and $9(n=3)$. A total of 20 sufficient laser shots were averaged per spectrum with a $200-\mu \mathrm{m}$ resolution in the spot raster. On average, a total of 
85 peaks were resolved on prostate tissue sections. A support vector algorithm (SVM) of 22 significant peaks resulted in a sensitivity and specificity of 85 and $91 \%$, respectively. A total of 4 candidate biomarker peaks with high impact on classification were detected: 2,753 and 6,704 Da for non-cancerous glands and 4,964 and 5,002 Da for cancerous glands. A second evaluation using a five-dimensional genetic algorithm resulted in a sensitivity and specificity of 70 and $84 \%$, respectively. However, the peaks were not identified.

In 2009, Cazares et al reported a case-control study designed to screen for differentially expressed proteins in prostate cancer (21). MSI profiles of tissue samples from 11 prostate carcinoma patients were compared with 11 healthy subjects. Samples were obtained prior to treatment and stored at $-80^{\circ} \mathrm{C}$. The model with the highest classification accuracy was constructed using 3 masses at $\mathrm{m} / \mathrm{z}$ 1,027, 4,274 and 4,355 and was capable of correctly classifying $85 \%$ of prostate tissue areas. Validation of an independent, blinded sample set ( 23 tumor and 31 benign sections) demonstrated this model's diagnostic potential with a correct classification of $81 \%$. Furthermore, the identification of the mitogen-activated protein kinase/extracellular signal-regulated kinase kinase kinase 2 at m/z 4,355 was immunohistochemically confirmed, distinguishing tumor from non-tumor tissues.

Lemaire et al reported MALDI-TOF imaging of 25 patients with ovarian cancer and 23 patients with benign tumors (16). Whereas Schwamborn and Cazares used frozen tissues, Lemaire et al compared tissue profiles of FFPE tissue samples in combination with on-tissue enzymatic digestion. The samples in the two studies were stored at $-80^{\circ} \mathrm{C}$. Among 100 detected individual peptide signals, one putative biomarker $(9,744 \mathrm{~m} / \mathrm{z})$ with a prevalence of $80 \%$ was determined by MSI. Subsequent identification by means of nanoESI MS/MS revealed a correspondence to 84 amino acid residues from the 11S proteasome activator complex, named PA28 or Reg- $\alpha$. Clinical validation was successfully carried out using Western blotting and immunohistochemistry. Groseclose et al screened an FFPE tissue microarray (TMA) comprised of duplicate needle core punches from 50 patients diagnosed with human non-small cell lung cancer and 10 adjacent normal lung punches (14). The TMA was subjected to on-tissue tryptic digestion followed by MSI. In this series, diagnostic models were developed using statistical classification models based on 73 peaks. This model classified the spectra from regions marked as adenocarcinoma by the pathologist with an accuracy of $98 \%$ and squamous cell carcinoma with an accuracy of $99 \%$. Characterization of the most prominent $\mathrm{m} / \mathrm{z}$ values identified 50 proteins (e.g., S100-A9, HSP $\beta$-1, Histone H2A) directly from the lung tumor TMA using MALDI-MS/MS sequence analysis.

Similary, Djidja et al published a report regarding novel tumor classification using MALDI-ion mobility separationMSI of a TMA (22). They used an independent sample set of 60 pancreatic adenocarcinoma needle cores from 30 patients (two spots from each cancer case) and 30 non-neoplastic needle cores (corresponding normal tissues). Principal component discriminant analysis was used, generating tumor classification models in respect to protein profile patterns. Several peptides could be identified and were statistically validated in other tissue cores and patient samples.
In 2010, Schwamborn et al published a set of 54 lymphatic tissue samples that were stored at $-80^{\circ} \mathrm{C}(23)$. They screened 32 tissues from patients with classical Hodgkin's lymphoma (CHL). Spectra of the malignancy samples were compared to those of 22 healthy volunteers with lymphadenitis. They utilized a Reflex IV MALDI-TOF-MS (Bruker) with a $200-\mu \mathrm{m}$ resolution for imaging experiments. As with their previous study, classification algorithms were developed and validated using bioinformatics tools, such as SVMs. Using the 24 most significant peaks, a classification of $84 \%$ sensitivity and $89 \%$ specificity was allowed. Out of these 24 peaks, 4 were of particular interest due to their higher impact on the classification: 4,736 and 4,746 Da for CHL, 4,962 and 5,000 Da for lymphadenitis. Additionally, a seven-dimensional genetic algorithm based on 7 peaks selected by hand due to greatest differential expression in the overall sum spectra resulted in an overall sensitivity and specificity of 85 and $87 \%$, respectively. Their masses ranged from 4.6 to $8.2 \mathrm{kDa}$ and contained the 4 masses from the SVM algorithm mentioned above.

The most recent article was published by Agar et al in April 2010 (24). A total of 20 tissue samples, including 8 cases of meningioma, 6 cases of glioma tumor samples and 6 non-tumor samples, were examined by MSI. The storage temperature was not specified. SVM for classification models combined 7 protein mass peaks to discriminate patient groups. However, neither a validation test-set nor an identification of the markers found was reported.

\section{MALDI-TOF imaging data relating to cancer}

This review of current literature regarding clinical applicability of MALDI-TOF imaging in oncology suggests further considerations. As shown in Table II, the accuracy of the MSI-based classification appears promising, although it is still dependent on sample collection procedures and pre-analytical conditions.

Regarding the latter, in 4 out of 7 studies, MSI was carried out on fresh-frozen tissue sections, therefore closely reflecting in vivo properties. However, the clear majority of clinical specimens stored in hospital tissue banks are FFPE, representing a large and valuable archive of diseased tissues. In our review, 3 out of 7 studies used sequence determination of tryptic fragments by MS/MS analysis directly after on-tissue digestion in FFPE specimens. However, the possibility that formalin stabilization of proteins by chemical cross-linking and subsequent antigen retrieval for MSI may mask valuable biomarkers or bring about false positives cannot be excluded. In this context, comparative studies running MSI on cryoconserved and corresponding FFPE tissue in parallel should be conducted to validate the use of FFPE archives. Until then, use of cryo-conserved tissues appears to be the superior approach for tissue proteome profiling in cancer. With increasing resolution, the quality of tissue samples may even increase in significance.

The highest clinical performances for MALDI-TOF MSI-based biomarker determination have been reported by combining information from several biomarkers in cluster analyses using, for example, SVMs. While the software packages supporting MSI analysis are user-friendly and as such highly appreciated, one should be aware that different project 


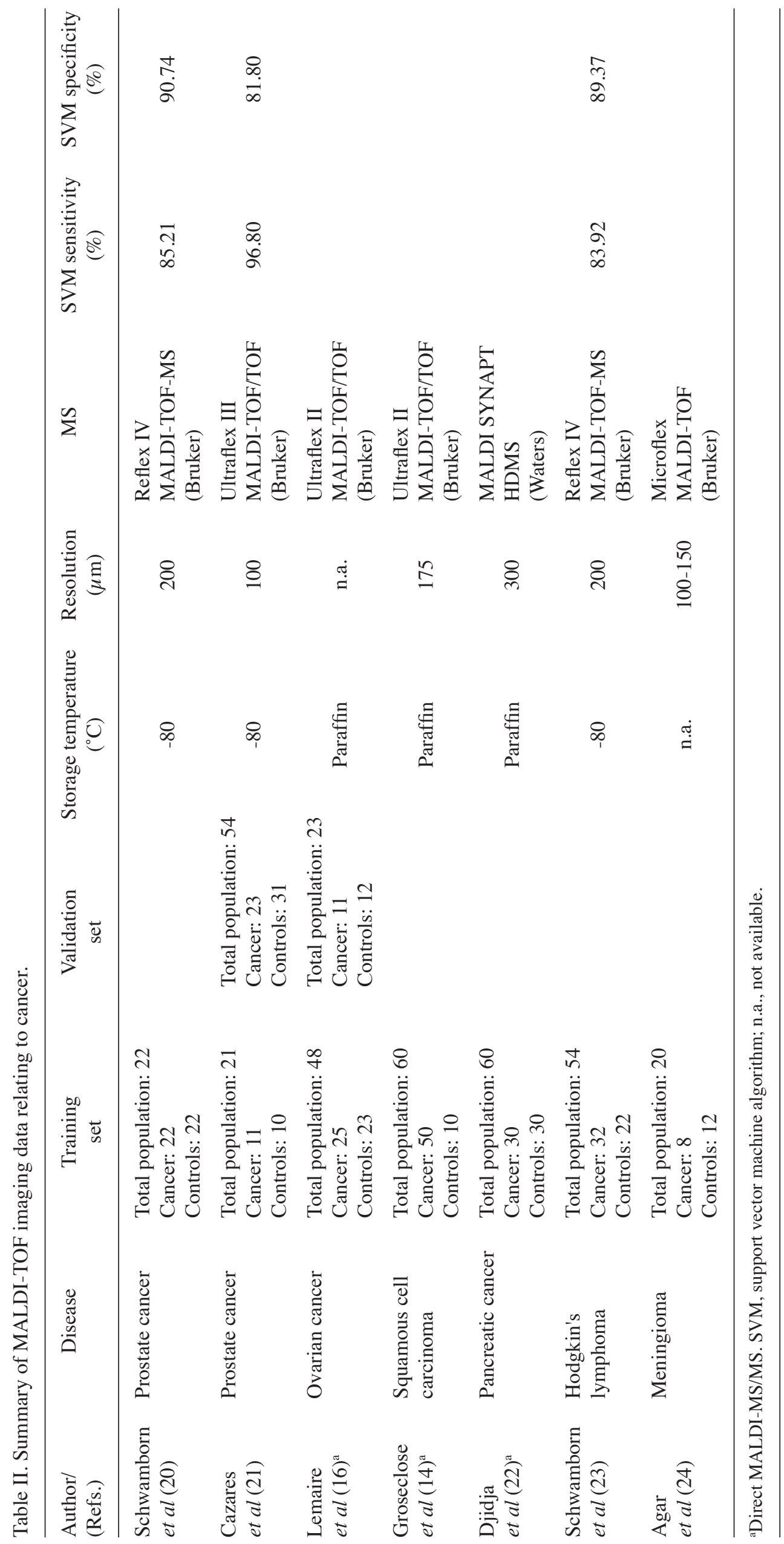


designs may require different bioinformatics approaches and that clinical application will require higher levels of quality control and application-tailored bioinformatics pipelines.

MSI proved its potential as a screening tool for tissue-based biomarker detection and peak picking classification approach. However, more emphasis should be placed on the identification of MSI-generated peak candidates and subsequent validation using clinically applicable techniques, such as ELISA and ECLIA in independent and large sample cohorts.

The majority of the reviewed studies did not choose study designs comprising a training and validation set. However, biomarker studies require large numbers of clinically well defined samples divided into training and validation sets, while the operator should work blinded for group affiliation of samples. Larger sample sets with 50 or more patient samples/ group should be preferred, particularly for validating newly identified markers. It should be mandatory for the reference/ control group to be derived from a cohort that is matched not only according to age and gender but also to sample storage duration and temperature. If not, this may introduce unanticipated and unrecognized bias unless the differences in handling are systematically investigated and shown not to affect the specific marker measurements (25-27).

The majority of the reviewed studies presented few or insufficient details on quality management of samples. Quality management of samples affords strict standard operation procedures (SOPs) regarding patient inclusion/ exclusion criteria, ethical permission and informed consent, sample collection, sample processing and sample storage until further use. In our experience and in light of other reports, sample preservation at temperatures of at least $-80^{\circ} \mathrm{C}$ is recommended even though it has been well recognized that certain biomarkers may continuously degrade during storage even at $-80^{\circ} \mathrm{C}(28-32)$. Therefore, if the stability of markers to be tested is not known or in case of screening studies for novel markers, sample storage in liquid/gaseous nitrogen $\left(-196^{\circ} \mathrm{C}\right)$ should be a prerequisite for any downstream analyses. In addition, shortand long-term storage temperature, freeze and thaw cycles and storage duration should be monitored and/or examined, since expression and degradation levels of the protein may change and therefore compromise clinical applicability. Degradation of biomarkers may be rapid and, more significantly, vary between different markers (33). Thus, the quality of the sample operating procedures will directly impact the quality and validity of the biomarker's result and its ultimate applicability to the patient. Given this context, we would like to stress the marked significance of detailed SOPs for sample management for proteomics analysis.

\section{Conclusion}

MALDI-TOF MSI offers a promising clinical prospect and can assist in detecting morphology-related biomarkers for improved personalized medicine. Due to the rapid acquisition of morphology-related proteomics patterns from complex biological samples, MSI shows advantages over conventional analytical techniques. Its strength lies in its independence from target-specific reagents such as antibodies, in its capability for direct identification of analytes (e.g., proteins and peptides) and for analysis of multiple analytes simultaneously.
With increasing resolution, evaluation of only a few cells and of low abundant proteins may be achievable.

Although current results are promising, future comprehensive, well defined screening and validation studies involving hundreds of samples are required to promote the translation of MALDI-imaging from bench to bedside.

\section{Acknowledgements}

This review study was performed by the Surgical Center for Translational Oncology - Lübeck (SCTO-L) and was supported by grants from the Werner and Klara KreitzFoundation (Kiel, Schleswig-Holstein), the H.W. and J. Hector-Foundation (Weinheim), the Ad Infinitum Foundation (Lübeck), and the North German Tumorbank of Colorectal Cancer (DKH \#108446).

\section{References}

1. Petricoin EF, Ardekani AM, Hitt BA, et al: Use of proteomic patterns in serum to identify ovarian cancer. Lancet 359: 572-577, 2002.

2. Alaiya A, Al-Mohanna M and Linder S: Clinical cancer proteomics: promises and pitfalls. J Proteome Res 4: 1213-1222, 2005.

3. Iwadate Y: Clinical proteomics in cancer research-promises and limitations of current two-dimensional gel electrophoresis. Curr Med Chem 15: 2393-2400, 2008.

4. Gorg A, Weiss W and Dunn MJ: Current two-dimensional electrophoresis technology for proteomics. Proteomics 4: 3665-3685, 2004.

5. O'Farrell PH: High resolution two-dimensional electrophoresis of proteins. J Biol Chem 250: 4007-4021, 1975.

6. Mannello F, Medda V and Tonti GA: Protein profile analysis of the breast microenvironment to differentiate healthy women from breast cancer patients. Expert Rev Proteomics 6: 43-60, 2009.

7. Gast MC, Schellens JH and Beijnen JH: Clinical proteomics in breast cancer: a review. Breast Cancer Res Treat 116: 17-29, 2009.

8. Zieske LR: A perspective on the use of iTRAQ reagent technology for protein complex and profiling studies. J Exp Bot 57: 1501-1508, 2006.

9. Qian WJ, Jacobs JM,Liu T, Camp DG II and Smith RD: Advances and challenges in liquid chromatography-mass spectrometrybased proteomics profiling for clinical applications. Mol Cell Proteomics 5: 1727-1744, 2006.

10. Fuchs B and Schiller J: MALDI-TOF MS analysis of lipids from cells, tissues and body fluids. Subcell Biochem 49: 541-565, 2008.

11. Habermann JK, Bader FG, Franke C, et al: From the genome to the proteome - biomarkers in colorectal cancer. Langenbecks Arch Surg 393: 93-104, 2008.

12. Ikonomou G, Samiotaki M and Panayotou G: Proteomic methodologies and their application in colorectal cancer research. Crit Rev Clin Lab Sci 46: 319-342, 2009.

13. Becker KF, Schott C, Hipp S, et al: Quantitative protein analysis from formalin-fixed tissues: implications for translational clinical research and nanoscale molecular diagnosis. J Pathol 211: 370-378, 2007.

14. Groseclose MR, Massion PP, Chaurand P and Caprioli RM: High-throughput proteomic analysis of formalin-fixed paraffinembedded tissue microarrays using MALDI imaging mass spectrometry. Proteomics 8: 3715-3724, 2008.

15. Groseclose MR, Andersson M, Hardesty WM and Caprioli RM: Identification of proteins directly from tissue: in situ tryptic digestions coupled with imaging mass spectrometry. J Mass Spectrom 42: 254-262, 2007.

16. Lemaire R, Desmons A, Tabet JC, Day R, Salzet M and Fournier I: Direct analysis and MALDI imaging of formalinfixed, paraffin-embedded tissue sections. J Proteome Res 6: 1295-1305, 2007.

17. Stauber J, Lemaire R, Franck J, et al: MALDI imaging of formalin-fixed paraffin-embedded tissues: application to model animals of Parkinson disease for biomarker hunting. J Proteome Res 7: 969-978, 2008. 
18. Kreeger PK and Lauffenburger DA: Cancer systems biology: a network modeling perspective. Carcinogenesis 31: 2-8, 2010.

19. Lagarrigue M, Becker M, Lavigne R, et al: Revisiting rat spermatogenesis with MALDI imaging at 20-microm resolution. Mo Cell Proteomics 10: M110 005991, 2011.

20. Schwamborn K, Krieg RC, Reska M, Jakse G, Knuechel R and Wellmann A: Identifying prostate carcinoma by MALDIImaging. Int J Mol Med 20: 155-159, 2007.

21. Cazares LH, Troyer D, Mendrinos S, et al: Imaging mass spectrometry of a specific fragment of mitogen-activated protein kinase/extracellular signal-regulated kinase kinase kinase 2 discriminates cancer from uninvolved prostate tissue. Clin Cancer Res 15: 5541-5551, 2009.

22. Djidja MC, Claude E, Snel MF, et al: Novel molecular tumour classification using MALDI-mass spectrometry imaging of tissue micro-array. Anal Bioanal Chem 397: 587-601, 2010.

23. Schwamborn K, Krieg RC, Jirak P, et al: Application of MALDI imaging for the diagnosis of classical Hodgkin lymphoma. J Cancer Res Clin Oncol 136: 1651-1655, 2010.

24. Agar NY, Malcolm JG, Mohan V, et al: Imaging of meningioma progression by matrix-assisted laser desorption ionization timeof-flight mass spectrometry. Anal Chem 82: 2621-2625, 2010.

25. Bonini P, Plebani M, Ceriotti F and Rubboli F: Errors in laboratory medicine. Clin Chem 48: 691-698, 2002.
26. Troyer D: Biorepository standards and protocols for collecting, processing, and storing human tissues. Methods Mol Biol 441: 193-220, 2008

27. Grantzdorffer I, Carl-McGrath S, Ebert MP and Rocken C: Proteomics of pancreatic cancer. Pancreas 36: 329-336, 2008.

28. Ericsson C, Peredo I and Nister M: Optimized protein extraction from cryopreserved brain tissue samples. Acta Oncol 46: 10-20, 2007.

29. Stacey GN and Day JG: Cryopreservation and freeze-drying protocols. Introduction. Methods Mol Biol 38: 1-5, 1995.

30. Wusteman MC, Pegg DE and Warwick RM: The banking of arterial allografts in the United Kingdom. A technical and clinical review. Cell Tissue Bank 1: 295-301, 2000.

31. McDonnell LA, Corthals GL, Willems SM, van Remoortere A, van Zeijl RJ and Deelder AM: Peptide and protein imaging mass spectrometry in cancer research. J Proteomics 73: 1921-1944, 2010.

32. Leiva IM, Emmert-Buck MR and Gillespie JW: Handling of clinical tissue specimens for molecular profiling studies. Curr Issues Mol Biol 5: 27-35, 2003.

33. Goodwin RJ, Dungworth JC, Cobb SR and Pitt AR: Timedependent evolution of tissue markers by MALDI-MS imaging. Proteomics 8: 3801-3808, 2008. 